\title{
THE EFFECT OF PNEUMOPERITONEUM AND POSITION ON THE OPTIC NERVE SHEATH DIAMETER DURING ROBOTIC RADICAL PROSTATECTOMY; AN ULTRASONOGRAPHIC EVALUTION
}

\author{
Atic E.', Cetingok H. ', Tok B.', Hergunsel G.O.', Yener Y.Z.' , Balkan B.'
}

'Bakirkoy Sadi Konuk Research and Tranining Hospital, Dept of Anaesthesiology \& Intensive Care, Istanbul, Turkey, ${ }^{2}$ Istanbul University, Dept of Anaesthesiology \& Pain Medicine, Istanbul, Turkey

\section{$\underline{\text { AIM }}$}

The deep trendelenburg position and pneumoperitoneum are applied in cases of Robot-Assisted Laparoscopic Radical Prostatectomy (RALRP) operations are thought to increase intracranial pressure. Orbital ultrasonography as a noninvasive measurement of the optic nerve sheath diameter (ONSD) have been reported in recent years in correlation with intracranial pressure. In this study, RALRP operations were done in different intra-abdominal pressures and the affect of the ONSD with different intra-abdominal pressure levels have been shown and research has been done to examine whether changes made in the cognitive functions have an impact on the patients.

\section{MATERIALS AND METHODS}

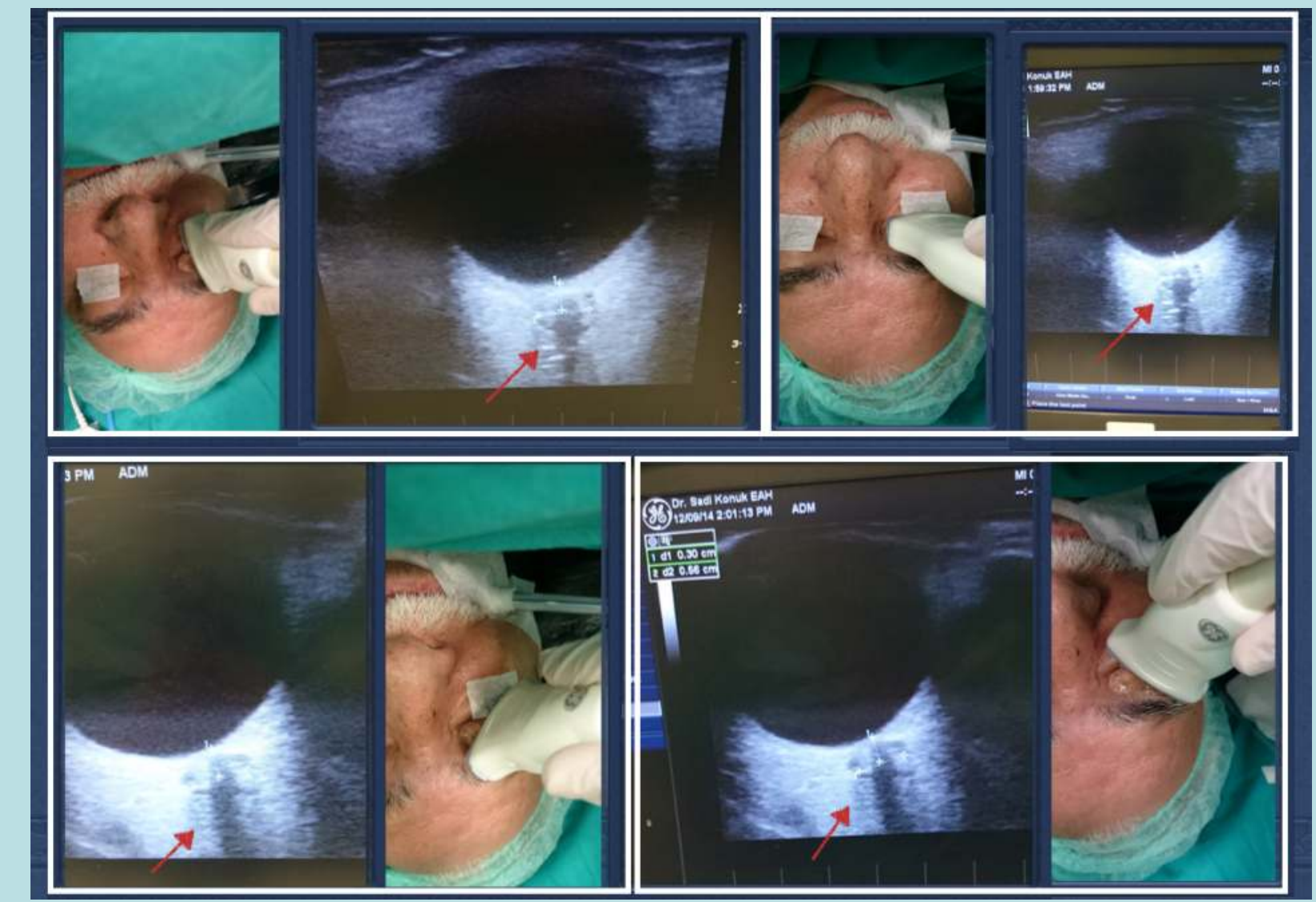

Fig.l. The ONSD measuring at 4 axis by ultrasound

RALRP operation arranged 40 patients to be included in this study whose ASA physical status was I-II and ages were 5070 included to this study. The patients were divided into 2 groups, Group I; with insufflation pressure as $10 \mathrm{mmHg}$ and Group 2; as $15 \mathrm{mmHg}$. The optic nerve sheath diameter measuring was done by ultrasound 10 times from the right eye of the patients with its 4 axis; before the induction of anesthesia, $5 \mathrm{~min}$ after induction, $5 \mathrm{~min}$ after abdominal insufflation, $5,15,30,60$ and $120 \mathrm{~min}$ of the trendelenburg position, $5 \mathrm{~min}$ after intra-abdominal insufflation and trendelenburg was terminated and 5 min after extubation was applied. Standardized Mini Mental State Examination (SMMSE) was performed I day before and 3 days later the operation.

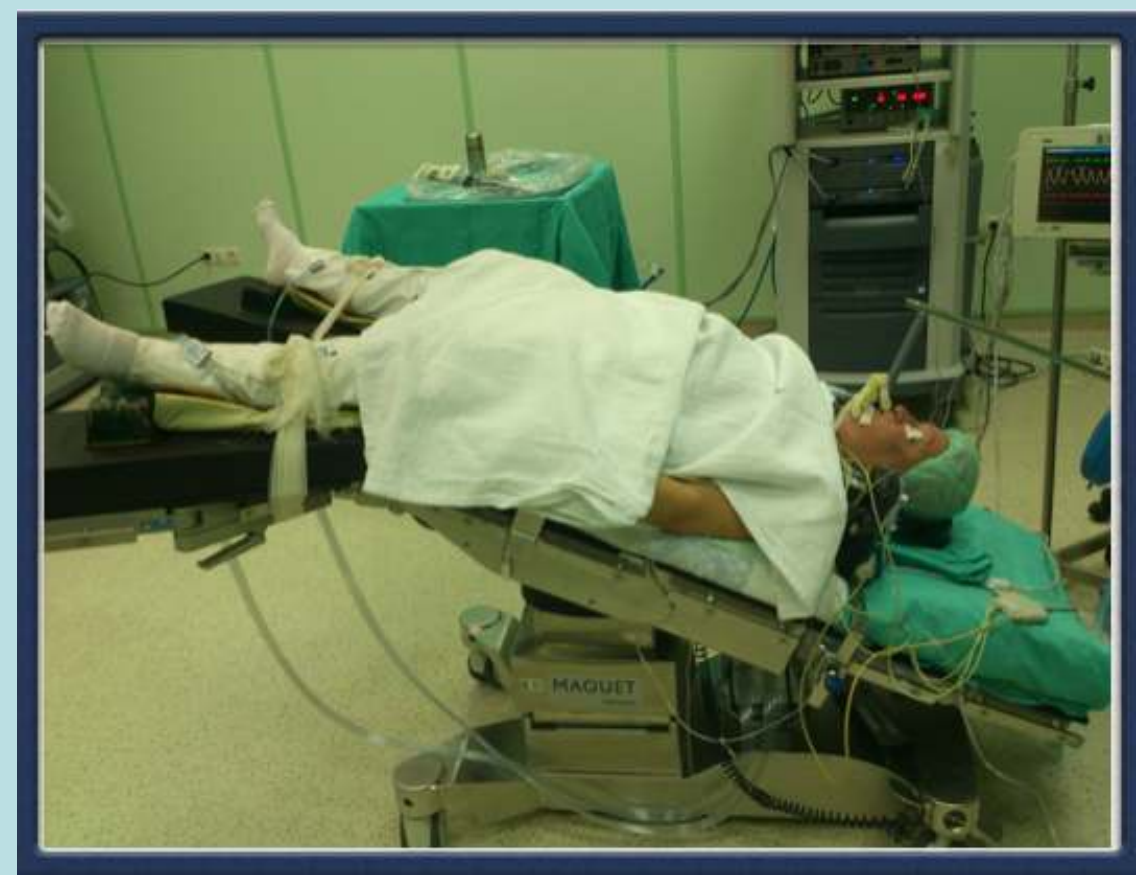

Fig. Il.Trendelenburg position during RALRP

\section{RESULTS}

There were no differences between the two groups for the operation time and demographical values. ONSD value was significantly $(p<0,05)$ lower in Group I at 60. and 120. min of the trendelenburg position and at the $5^{\text {th }}$ min of returning to supine position. According to SMMSE results for the two groups, there were no difference between preop and postop. ean airway pressure and ONSD values in deep trendelenburg position during operation increased in both groups compared with baseline values; but decreased back to levels similar to their baseline values at the end of the operation parameters with the supine position.

\section{CONCLUSION}

These results determined that lower intracranial pressure occurs at the low insufflation group by lower ONSD values with the prolongation of the deep trendelenburg position, but this difference did not make a significant difference in terms of cognitive function, which is viewed by the SMMSE.

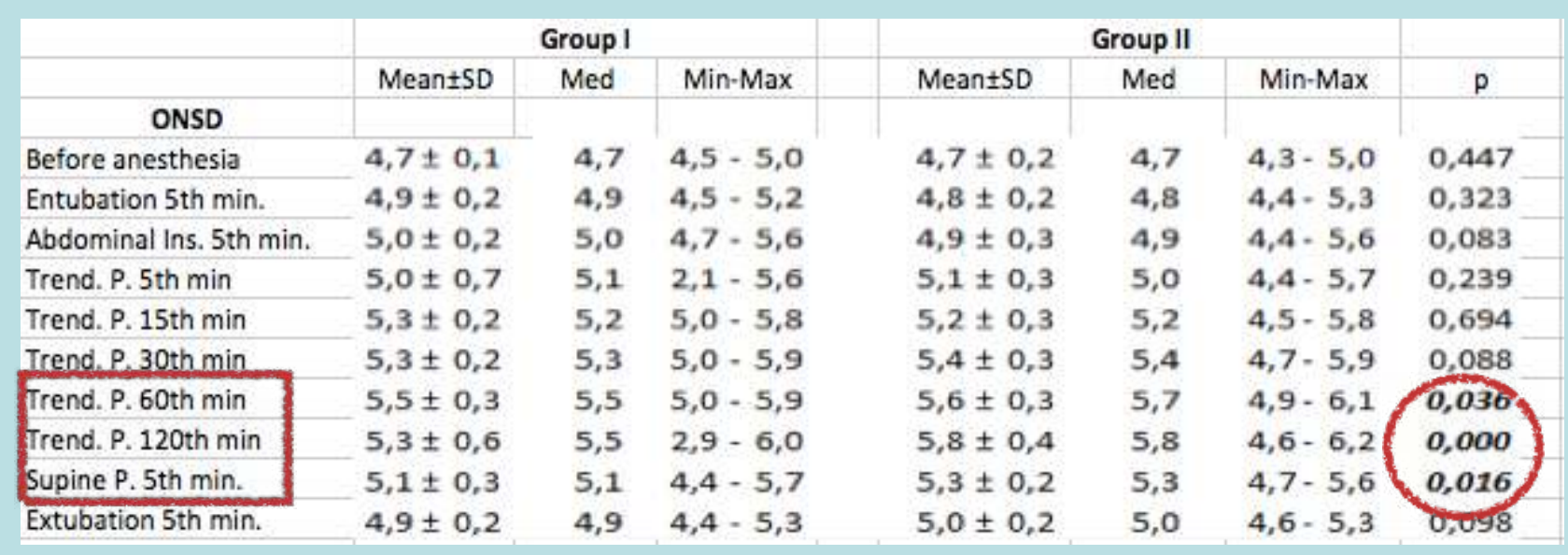

Table I. Comparision of ONSD values for two groups 